ISSN 2519-7398 (Versión electrónica)

DOI: http://dx.doi.org/10.21704/ac.v78i2.1057

(C) Universidad Nacional Agraria La Molina, Lima - Perú

\title{
Determinación de la demanda hídrica del cultivo de quinua QML01 (Chenopodium Quinoa Willd) en la Molina
}

\author{
Water demand of the QLM01 quinoa crop (Chenopodium Quinoa Willd) in la Molina
}

\author{
Jerónimo García Villanueva ${ }^{1 *}$; Jhon Huahuachampi²; Leonel Soto ${ }^{3}$
}

*Autor de correspondencia

\begin{abstract}
Resumen
El propósito de esta investigación fue determinar la demanda hídrica (Em) y el coeficiente Kc del cultivo de quinua. Este cultivo, es de alto valor alimenticio y nutritivo, actualmente es sembrado en condiciones agroclimáticas de costa y sierra, donde el agua para riego es un recurso escaso, siendo necesario hacer un uso racional de este recurso, con el fin de conservar el suelo, obtener buena producción y óptimo rendimiento. La determinación de la Em y Kc, fue evaluado para cada una de las cuatro etapas de desarrollo de la quinua QLM01. Para la determinación del Em, se instaló y operó un lisímetro de nivel freático constante; para determinar el $\mathrm{Kc}$, se utilizaron la evapotranspiración de referencia (ETo) que fue estimado con el método Penman-Monteith y método Penman adaptado para el Perú, así como la evapotranspiración máxima (Em). Los resultados indican que la quinua QLM01 requiere un volumen de $2623 \mathrm{~m}^{3} / \mathrm{ha}$ para una campaña; siendo el requerimiento de agua para la etapa de inicio, desarrollo, mediados de temporada y etapa Final 377, 588, 957 y $699 \mathrm{~m}^{3} / \mathrm{ha}$, respectivamente. Los coeficiente Kc hallados para la quinua QLM01 fueron respectivamente de 0.554, 0.98, 1.271 y 0.904 para las etapas del cultivo. Información fundamental para implementar diversos sistemas de cultivo agrícola.
\end{abstract}

Palabras claves: demanda hídrica; coeficiente Kc; evapotranspiración máxima; evapotranspiración de cultivo; evapotranspiración de referencia.

\begin{abstract}
Abstrac
The purpose of this research was to determine the water demand (Em) and the Kc coefficient of the quinoa crop. This crop, is of high nutritional and nutritive value, is currently planted in agroclimatic conditions of coast and sierra, where water for irrigation is a scarce resource, being necessary to make a rational use of this resource, in order to conserve the soil, obtain good production and optimum performance. The determination of Em and Kc was evaluated for each of the four stages of QLM01 quinoa development. For the determination of Em, a constant water level lysimeter was installed and operated; to determine the Kc, we used the reference evapotranspiration (ETo) that was estimated using the Penman-Monteith method and Penman method adapted for Peru, as well as the maximum evapotranspiration (Em). The results indicate that quinoa QLM01 requires a volume of $2623 \mathrm{~m}^{3}$ / ha for one season; being the water requirement for the beginning, development, mid-season and final stages 377, 588, 957 and $699 \mathrm{~m}^{3} /$ ha, respectively. The coefficients Kc found for quinoa QLM01 were respectively $0.554,0.98,1.271$ and 0.904 for the culture stages. Key information for implementing various agricultural cropping systems.
\end{abstract}

Key words: water demand, Kc crop coefficient, potential evapotranspirations, crop evapotranspirations, reference evapotranspirations.

\section{Introducción}

La quinua es un cultivo de alto valor alimenticio y nutritivo con una adaptabilidad a diferentes condiciones agroclimáticas. En el país su cultivo se lleva a cabo en la costa y Sierra, por lo general en la sierra son cultivos de secano y según Mujica et al. (2001) estos cultivos prosperan con precipitación anual que oscila entre $250-$ $500 \mathrm{~mm}$, mientras en la costa su cultivo es bajo riego; su productividad es mayor en la costa que en la sierra la cual depende de la variedad, disponibilidad de agua, tecnología empleada, manejo fitosanitario, entre otros factores, así por ejemplo SENASA (2014) indica un rendimiento de $5.5 \mathrm{tn} /$ ha para las variedades Salcedo INIA y El Altiplano pero que son muy susceptibles al mildiu; el IICA (2015) presenta el rendimiento de este cultivo por departamentos de donde extraemos la Tabla 1 para el año 2014 en la cual se observa mayor rendimiento en aquellos departamentos que poseen áreas agrícolas destinados a este cultivo en la costa, pero 
en aquellos departamentos con campos agrícolas sólo en los andes los rendimientos están por debajo de los de costa; según la misma fuente, el rendimiento promedio del país para 2014 fue de $1.68 \mathrm{tn} / \mathrm{ha}$. La mayor producción en la costa se debe al uso de la tecnología sobre todo el riego tecnificado, la mayoría de las empresas agrícolas usan el riego tecnificado por goteo, por aspersión, entre otras.

En la agricultura bajo riego es necesario tener en cuenta el uso sustentable del recurso suelo y agua, en la costa peruana el recurso agua es deficitario por lo que es imperativo su uso racional en las prácticas del riego aplicando la cantidad necesaria y en el tiempo oportuno; una aplicación más de lo necesario implica deterioro del recurso suelo y una aplicación menos de lo necesario constituye disminución de la producción del cultivo debido a la reducción de la actividad fotosintética de la planta. Conocer la cantidad necesaria de agua para la aplicación del riego implica determinar la demanda o requerimiento hídrico del cultivo en consideración de la fase fenológica en que se encuentra. La demanda hídrica de los cultivos (ETc) es el consumo de agua o evapotranspiración del cultivo con el suelo sin deficiencia de agua desarrollándose en condiciones controladas de sanidad y fertilidad, en forma directa se determina experimentalmente haciendo uso de lisímetros y a falta de estos se determina haciendo uso de la evapotranspiración de referencia (ETo) y el coeficiente hídrico Kc del cultivo en consideración; bajo esa condiciones debido a que el suelo está en todo momento en condiciones óptimas de humedad la evapotranspiración tiene su valor más alto y por ello también se le denomina como evapotranspiración máxima (Em). Estudios de Kc del cultivo de quinua existen pocos, Choquecallata et al. (1991) determinaron la evapotranspiración del cultivo de quinua por disimetría y su relación con la evapotranspiración potencial en el Altiplano Boliviano cuyo resultado de Kc se muestra en Tabla 2. cita resultado de Kc determinado por INIA para 6 estados fenológicos de la quinua cuyos valores son 0.3 para 4 hojas verdaderas, 0.5 para 6 hojas verdaderas, 0.8 para ramificación, 1.0 para floración, 0.9 para grano lechoso y 0.75 para grano pastoso. Los cálculos de la ETc son importantes en el monitoreo de las necesidades de agua con fines de determinación de la lámina de aplicación de riego; pero vemos que depende mucho del Kc que se utiliza para ello; las revisiones de los trabajos desarrollados sobre Kc de la quinua muestran diferencias entre ellos siendo más resaltante esas diferencias en la etapa de floración y formación de granos, las cuales son las fases críticas para la obtención de mayor o menor rendimiento del cultivo, son en esas fases el cultivo debe encontrarse en condiciones óptimas de humedad.

Por todo lo manifestado, en este trabajo se plantearon los siguientes objetivos:

a) Determinar experimentalmente la evapotranspiración máxima del cultivo

b) Determinar los Kc del cultivo.

\section{Materiales y métodos}

El experimento se llevó a cabo en el campo experimental del Instituto Nacional de Innovación Agraria (INIA) de La Molina, ubicado geográficamente en la latitud $12.085^{\circ} \mathrm{S}$, longitud $76.951^{\circ} \mathrm{W}$ y una altitud de $251 \mathrm{~m}$. El campo asignado para el cultivo fue de $84 \mathrm{~m}^{2}$.

El equipo de lisímetro constituido por tres tanques de fibra de vidrio (un tanque de cultivo, un tanque controlador del nivel freático y un tanque alimentador de agua con sistema de lectura en base a una pipeta), para su implementación e instalación requirió de accesorios y materiales como se indican a continuación:

Tabla 1. Rendimiento de quinua para el año 2014 en algunos departamentos del país

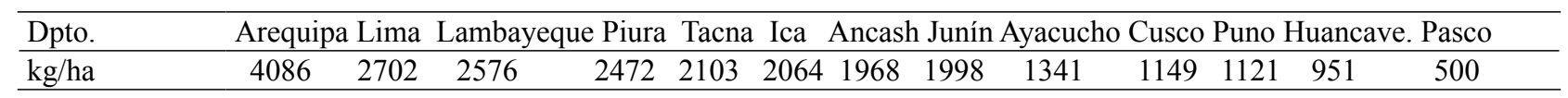

Tabla 2. Evapotranspiración máxima (Em) lisimétrica, ETo método de Penman y Kc de la quinua Patacamaya (1989/1990).

\begin{tabular}{lllll}
\hline Periodo & Em (mm/día) & ETo(mm/día) & Kc & Fase fenológica \\
\hline $15 / 12 / 89-17 / 12 / 89$ & 2.8 & 4.85 & 0.58 & Cuatro hojas verdaderas \\
$05 / 01 / 90-10 / 01 / 90$ & 2.77 & 4.40 & 0.63 & Inicio de panojamiento. \\
$11 / 01 / 90-16 / 01 / 90$ & 3.12 & 4.25 & 0.73 & Panojamiento \\
26/01/90-31/01/90 & 3.32 & 3.68 & 0.90 & Inicio de floración \\
$03 / 02 / 90-11 / 02 / 90$ & 4.21 & 4.16 & 1.01 & Floración o antesis \\
$13 / 02 / 90-20 / 02 / 90$ & 4.54 & 4.19 & 1.08 & Floración o antesis \\
$01 / 03 / 90-08 / 03 / 90$ & 4.71 & 4.12 & 1.14 & Inicio de grano lechoso \\
$10 / 03 / 90-19 / 03 / 90$ & 4.08 & 4.06 & 1.00 & Fin de grano lechoso \\
23/03/90-05/04/90 & 3.25 & 4.17 & 0.78 & Grano pastoso \\
Fuente: Choquecallata et al. (1991) & & & &
\end{tabular}

Por otro lado, Geerts (2008) en cultivo de quinua sembrado en surcos, también en el Altiplano Boliviano, encontró Kc promedio de $0.6,1.33$ y 0.6 en la etapa inicial, medio y final respectivamente; para caso del Perú Mori (2015)
Accesorios: pipeta de $50 \mathrm{ml}, 2$ tubos de rosca de PVC de $1 / 2$ “ (diámetro de tubos utilizados en los tanques), 6 unidades de codos, $1 \mathrm{~T}, 1$ unión universal, una tapa de tubo todos de $1 / 2$ “, una boya con su sistema de válvula para 
fijar en el orificio lateral superior del tanque controlador de nivel freático, $10 \mathrm{~m}$ de manguera reforzada de $3 / 4$ “ con cuatro abrazaderas, un frasco de pegamento de $1 / 8$ y cuatro unidades de teflón.

Materiales: 10 bolsas de cantos rodados de dos a cuatro pulgadas de diámetro $(5 \mathrm{a} 10 \mathrm{~cm}), 10$ bolsas de grava de 0.5 a $1.5 \mathrm{~cm}$ de diámetro, 10 bolsas de piedra chancada de techo (uno a dos $\mathrm{cm}$ de diámetro). Por otro lado, para la determinación de los parámetros físicos del suelo como humedad en capacidad de campo y densidad global, se requirió de anillos muestreadores, balanza digital y estufa.

\section{Datos meteorológicos}

Para la determinación de la evapotranspiración de referencia se utilizó datos de horas de sol, temperatura máxima, mínima, viento y humedad relativa del año 2015 de la Estación Alexander Von Humboldt.

\section{Determinación de la demanda hídrica del cultivo}

La determinación de la evapotranspiración del cultivo (ETc) o evapotranspiración máxima $(\mathrm{Em})$ de quinua se realizó directamente a través de la lectura, del consumo de agua, en el tanque alimentador del lisímetro; para la obtención de la lámina de agua evapotranspirada por el cultivo se utilizó la siguiente expresión:

$$
E m=\left(\frac{\mathrm{ra}}{\mathrm{rc}}\right)^{2} \cdot L(\mathrm{ml})
$$

donde $r a$ y $r c$ son el radio del tanque alimentador y tanque de cultivo respectivamente y $L$ es la diferencia entre la lectura final e inicial del sistema de lectura del lisímetro en el intervalo de tiempo en consideración (12 horas y/o 24 horas) y está expresado en mililitros $(\mathrm{ml})$; como la evapotranspiración del cultivo se expresa en milímetros ( $\mathrm{mm} /$ día), es necesario convertir la medida volumétrica (ml) a medida lineal $(\mathrm{mm})$, para la pipeta utilizado la equivalencia fue $2 \mathrm{ml}=11.5 \mathrm{~mm}$ con lo cual se tiene la ecuación (2.2):

$$
E m=\left(\frac{r a}{r r}\right)^{2} 5.75 L(\mathrm{~mm})
$$

Además en un lisímetro ya instalado y calibrado el radio de los tanques utilizados son constantes y para este caso fueron $r a=25.5 \mathrm{~cm}$ y $r c=57 \mathrm{~cm}$, remplazando en (2.2) se obtuvo el factor de corrección (fc) con lo cual la Em está dada por la expresión (2.3):

$$
\mathbf{E m}=\mathbf{1 . 1 6 8 9 2} \mathbf{L} \quad(\mathrm{mm} / \mathrm{dí}) \quad(2.3)
$$

Es preciso indicar que la evapotranspiración diaria está dada por la suma Em del periodo diurno y del periodo nocturno. El proceso de lectura del lisímetro se realizaron diariamente a las 7 y 19 horas desde la siembra del cultivo hasta su maduración.

\section{Instalación y calibración del lisímetro}

Una vez fijado el emplazamiento del equipo de lisímetro y por ende del tanque de cultivo se procedió a su calibración; la cual consistió en establecer la capa de suelo correspondiente a la zona radicular $(0-25 \mathrm{~cm})$ a una humedad en capacidad de campo; para ello, una vez que la humedad llegara a la superficie, se tomó muestras de suelo para determinar humedad gravimétrica y compararla con la humedad en capacidad de campo determinada previamente en el campo experimental; el tanque controlador de nivel freático se sube o baja según la humedad encontrada es menor o mayor a la capacidad de campo, el proceso se repite hasta conseguir se aproxime a la humedad en capacidad de campo. Mayor detalle sobre el tema se presenta en anexo.

\section{Determinación del coeficiente Kc del cultivo}

El Kc del cultivo está en función de la evapotranspiración de referencia (ETo) y la evapotranspiración del cultivo (Etc) expresado mediante la siguiente relación:

$$
K C=\frac{E T C}{E T O}
$$

ETc es la evapotranspiración del cultivo de quinua, determinado en forma diaria durante el periodo vegetativo del cultivo a través de la lectura del lisímetro de nivel freático constante.

Para el cálculo de la ETo se utilizó la ecuación de FAO Penman-Monteith, Allen et al. (2006) y el método de Penman modificado para condiciones del Perú Garcia (1992), las expresiones utilizadas en cada uno de los métodos se indican a continuación.

\section{Ecuación de FAO Penman-Monteith}

Este método es actualmente el recomendado para el cálculo de la evapotranspiración de referencia (ETo) debido a que contiene todas las variables climáticas que controlan la evapotranspiración de referencia; sin embargo su utilización se ve restringida por la falta de estaciones climatológicas que registran todas las variables climáticas requeridas por la ecuación sobre todo viento y humedad. Su expresión es la siguiente:

$$
E T o=\frac{0.408 \Delta(R n-G)+\gamma \frac{900}{T+273} U 2(e s-e a)}{\Delta+\gamma(1+0.34 U 2)}
$$

Donde:

ETo, evapotranspiración de referencia $\left(\mathrm{mm} \mathrm{día}^{-1}\right)$

$\mathrm{Rn}$, radiación neta en la superficie del cultivo $\left(\mathrm{MJ} \mathrm{m}^{-2} \mathrm{dí}^{-1}\right)$

$\mathrm{Ra}$, radiación extraterrestre $\left(\mathrm{mm} \mathrm{día}^{-1}\right)$

$\mathrm{G}$, flujo del calor de suelo $\left(\mathrm{MJ} \mathrm{m}^{-2} \mathrm{día}^{-1}\right)$

$\mathrm{T}$, temperatura media del aire a $2 \mathrm{~m}$ de altura $\left({ }^{\circ} \mathrm{C}\right)$

$\mathrm{U} 2$, velocidad del viento a $2 \mathrm{~m}$ de altura $\left(\mathrm{m} \mathrm{s}^{-1}\right)$

es, presión de vapor de saturación $(\mathrm{kPa})$

ea, presión real de vapor de agua $(\mathrm{kPa})$

es - ea, déficit de saturación de presión de vapor $(\mathrm{kPa})$

$\Delta$, pendiente de la curva de presión de saturación de vapor de agua $(\mathrm{es}=\mathrm{ea})\left(\mathrm{kPa} /{ }^{\circ} \mathrm{C}\right)$ 
$\gamma$, constante psicrométrica $\left(\mathrm{kPa}^{\circ} \mathrm{C}^{-1}\right)$.

El flujo de calor $\mathrm{G}$ para cálculos diarios es aproximadamente cero; la radiación neta $\mathrm{Rn}$ está dada como la diferencia entre la radiación neta en onda corta (Rns) y la radiación neta en onda larga $(\mathrm{Rnl})$ y está en $\mathrm{MJ} \mathrm{m}^{-2}$ día ${ }^{-1}$ y para expresar en $\mathrm{mm}$ día ${ }^{-1}$ se aplicó el factor de conversión 0.408 , las ecuaciones 2.6 y 2.7 se utilizaron para sus cálculos respectivos: $R n s=(1-\alpha) R s \quad\left[\mathrm{MJ} \mathrm{m}^{-2}\right.$ día $\left.^{-1}\right]$

$\left.R n l=\sigma\left[\left(\operatorname{TmaK}^{4}+\operatorname{TmiK}^{4}\right) / 2\right)\right](0.34-0.14 \sqrt{ } \mathrm{ea})[1.35(\mathrm{Rs} / \mathrm{Rso})-$ $0.35)]\left[\mathrm{M} . \mathrm{Jm}^{-2} \cdot \mathrm{dí}^{-1}\right] \quad(2.7)$

En las que el valor de la constante Stefan y Boltzman $\sigma$ es igual a $4.903 \times 10^{-9} \mathrm{MJ} \mathrm{K} \mathrm{K}^{-4} \cdot \mathrm{m}^{-2} \cdot$ día $^{-1}$, Rs radiación solar incidente en la superficie fue calculado con la ecuación 2.8 , en la cual la radiación extraterrestre Ra está dada por la ecuación 2.9, además Rso radiación solar en la superficie en un día despejado se calcula con la ecuación 2.10, las cuales se presentan a continuación:

$R s=k_{R S}(T m a-T m i)^{0.5}\left[\mathrm{MJ} \mathrm{m}^{-2} \mathrm{dí}^{-1}\right]$

En la ecuación $2.8 \mathrm{k}_{\mathrm{RS}}$ es el coeficiente de ajuste cuyo valor adoptado fue 0.19 debido a que la localidad del campo experimental está cercano al mar, además es necesario indicar que Tma y Tmi son la temperatura máxima y mínima del día.

$\mathrm{a}=(24 \mathrm{x} 60 / \pi)^{*} G s \cdot d r[W s \cdot \sin (\varphi) \sin (\delta)+\cos (\varphi) \cos (\delta) \sin (W s)]$ $\left[\mathrm{MJ} \mathrm{m}{ }^{-2}\right.$ día $\left.^{-1}\right]$

en las que la distancia relativa inversa Tierra-Sol (dr), el ángulo de declinación solar $(\delta)$ y ángulo horario de puesta del sol (Ws) están dados por las ecuaciones 2.11, 2.12 y 2.13 respectivamente.

$d r=1+0.033 \cos [(2 \pi / 365) \mathrm{J}](2.11)$ el número de días del año (J) varía de 1 el primero de enero a 365 el 31 de diciembre. $\delta=0.409 \sin [(2 \pi / 365) \mathrm{J}-1.39]$ [radianes] (2.12)

$W_{s}=\arccos (-\operatorname{tg}(\varphi) \cdot \operatorname{tg}(\delta))$ [radianes $](2.13)$

$R s o=\left(0.75+2 \times 10^{-5} Z\right) R a\left[\mathrm{MJ} \mathrm{m}^{-2} \mathrm{dí}^{-1}\right] \quad$ (2.10)

en la ecuación $2.10, \mathrm{Z}$ es la altitud del lugar expresado en metros $(\mathrm{m})$; además es preciso aclarar que $(\varphi)$ es la latitud del lugar positivo para hemisferio norte y negativo para hemisferio sur y que Gs es la constante solar igual a 0.082 $\mathrm{MJ} \mathrm{m} \mathrm{min}^{-1}$.

Otras expresiones necesarias para determinar la ETo son las siguientes:

$e_{a}=0.6108 \mathrm{exp}(17.27 \mathrm{Tmi} / \mathrm{Tmi}+237.3)[\mathrm{kPa}]$

$e_{s}=[(\mathrm{e}(\mathrm{Tma})+\mathrm{e}(\mathrm{Tmi}) / 2][\mathrm{kPa}]$

$\Delta=\frac{4098\left[0.6108 \cdot \exp \left(\frac{17.27 \mathrm{~T}}{\mathrm{~T}+237.3}\right)\right]}{(\mathrm{T}+237.3)^{2}}\left[\mathrm{kPa}^{\circ} \mathrm{C}^{-1}\right]$

$\gamma=(\mathrm{Cp} . \mathrm{P} / \varepsilon . \lambda)\left[\mathrm{kPa}^{\circ} \mathrm{C}^{-1}\right]$

en la cual $\mathrm{Cp}=1.013 \times 10^{-3} \mathrm{MJ} \mathrm{kg}^{-1}{ }^{\circ} \mathrm{C}^{-1}, \varepsilon=0.622$ y $\lambda=2.45$ $\mathrm{kPa}{ }^{\circ} \mathrm{C}^{-1}$

$\mathrm{U}_{2}=\mathrm{U}_{\mathrm{Z}}\left[4.87 / \ln \left(“ 67.8 \mathrm{Z}\right.\right.$ " -"5.42” )] $\left[\mathrm{m} \mathrm{s}^{-1}\right]$, donde $\mathrm{Uz}$ es la velocidad del viento a la altura $Z$ y $Z$ es la altura de instalación del anemómetro.

$\mathrm{P}=101.3[(293-0.0065 \mathrm{Z}) / 293] 5.26[\mathrm{kPa}]$, siendo $\mathrm{Z}$ altitud del lugar en metros $(\mathrm{m})$.

\section{Ecuación de Penman modificado para Perú}

$E T o=E o=\frac{\frac{m \cdot e_{s}}{P T^{2}}(R n)+E a}{\frac{m \cdot e_{s}}{P \cdot T^{2}}+1}\left(\frac{m m}{\text { día }}\right) R n$

$=$ Rnoc + Rnol, Rnoc $=Q i(1-\underline{\alpha})$

Donde:

$\mathrm{m}$ : $8.03 \times 10^{6}\left({ }^{\circ} \mathrm{K}^{2}\right)$, calculado con $\mathrm{L}(584.9 \mathrm{cal} / \mathrm{gr})$ para temperatura media de la superficie evaporante de $22^{\circ} \mathrm{C}$.

$\mathrm{e}_{\mathrm{s}}$ : Presión de saturación del aire a la temperatura absoluta $\mathrm{T}$ dada por la expresión

$\mathrm{e}_{\mathrm{s}}=9.422 \times 10^{23} \cdot \mathrm{T}^{-5.08} \cdot \exp (-6801.27 / \mathrm{T}) \quad(\mathrm{mb})$

P: Presión atmosférica promedio a nivel de estación, para las condiciones topográficas del Perú, puede estimarse en función de la altitud $\mathrm{Z}$ expresado en metros con la siguiente expresión:

$\mathrm{P}=1014.78 . \exp \left(-1.17 \times 10^{-4} \mathrm{Z}\right)(\mathrm{mb})$

$\mathrm{Rn}$ : es la radiación neta expresado en $\mathrm{mm} / \mathrm{día}$ ( $\mathrm{Rn}$ $=$ Ronc + Rnol)

$\mathrm{T}$ : temperatura absoluta del sistema (puede sustituirse con la temperatura de caseta

Ea: es el término Aerodinámico, para la costa central del país está dado por $(-0.53+1.4 \mathrm{Rnol})(\mathrm{mm} /$ día $)$ y la radiación neta en onda larga, Rnol, para la costa central se determinó con la expresión

Rnol $=(-0.24+0.23 \Delta \mathrm{T})(\mathrm{mm} /$ día $)$

en la cual $\Delta \mathrm{T}=\mathrm{Tma}-\mathrm{Tmi}$ es el rango diurno de la temperatura.

La radiación neta en onda corta, para la costa central, se determinó con la expresión

Rnoc $=$ Qi $(1-\alpha)=$ Qs $[0.06+0.64(\Delta \mathrm{T} / \mathrm{N})](1-\alpha)$ $(\mathrm{mm} /$ día $)$

en la cual se consideró el albedo $(\alpha)$ igual a 0.21 y la radiación en el tope de la atmósfera (Qs) al igual que la duración del día solar $(\mathrm{N})$ están dadas por las expresiones 2.9 (multiplicado por 0.41 ) y 2.13 respectivamente.

Una vez determinado la evapotranspiración de referencia (ETo) por cada uno de los métodos se procedió a determinar el Kc diario durante todo el periodo vegetativo y luego se realizó el análisis de su variación temporal para ambos métodos.

\section{Resultados y discusiones}

\section{Determinación de la demanda hídrica del cultivo}

La siembra del cultivo de quinua, variedad QLM01, en el lisímetro se llevó a cabo el 22/08/2015 y a los 4 días se 
dio el 100\% de emergencia. Es así desde el 22 de agosto comenzó a operar el lisímetro y el consumo de agua o evapotranspiración máxima (Em) del cultivo cuantificado a nivel diario se muestra en la Figura 1 y está representado por la línea de asteriscos; se observa el comportamiento temporal diario es oscilante pero con clara tendencia de aumento desde la etapa inicial a mediados de emporada a partir del cual la tendencia es de disminución, los valores extremos mínimo de $0.8 \mathrm{~mm} /$ día se registró en la etapa inicial y el máximo de $5.4 \mathrm{~mm} /$ día en la etapa de $100 \%$ de desarrollo vegetativo denominado también como mediados de temporada (Allen et al., 2006). La Figura también muestra la evapotranspiración de referencia (ETo) para método de Penman Monteith (PenMonteith) y Penman adaptado para el Perú (PenModif) Garcia (1990), al comparar el comportamiento temporal de Em y ETo se distingue etapas durante el cual Em es menor a ETo, otra etapa en la cual ambas son aproximadamente iguales y otra en la cual Em mayormente es mayor a Eto, tal característica nos permite agrupar en cuatro etapas el ciclo vegetativo del cultivo como se observa en al Tabla 3, esas etapas son denominados como Inicio(I), Desarrollo (II), Mediados de temporada (III) y Final de temporada (IV) (Allen et al, 2006); la Tabla indicada muestra la demanda hídrica de $37.7 \mathrm{~mm}, 58.8 \mathrm{~mm}, 95.73 \mathrm{~mm}$ y $69.9 \mathrm{~mm}$, respectivamente, en cada una de esas etapas y el total de $262.3 \mathrm{~mm}$ en el periodo vegetativo del cultivo. Por otro lado, con la finalidad de visualizar en forma más clara las etapas indicadas se procedió a reducir las oscilaciones determinando el promedio cada tres días cuyo resultado se muestra en la Figura 2, la cual muestra las característica ya indicadas y con mayor claridad.

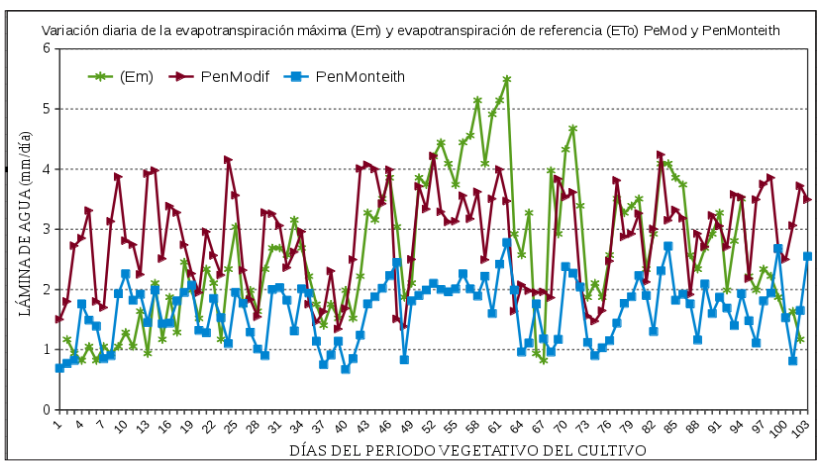

Figura 1. Variación temporal diaria de la demanda hídrica (Em) del cultivo de quinua y evapotranspiración de referencia (ETo) por método Penman Monteith (PenMonteith) y Penman adaptado para el Perú (PenModif)

El lisímetro de nivel freático constante proporciona agua al sistema cultivo en forma continua y en ningún momento existe déficit de disponibilidad de agua para el proceso de evapotranspiración, entonces el comportamiento temporal de aumento de Em obedece al aumento de los órganos evpotranspirantes y fotosintetizantes, el cual ocurre solo hasta que el cultivo llegue al $100 \%$ de su desarrollo (cultivo completamente desarrollado), posterior a ello empieza la senescencia de las hojas con las que demanda hídrica también empieza a disminuir la cual se acentuará posteriormente debido al proceso de maduración.

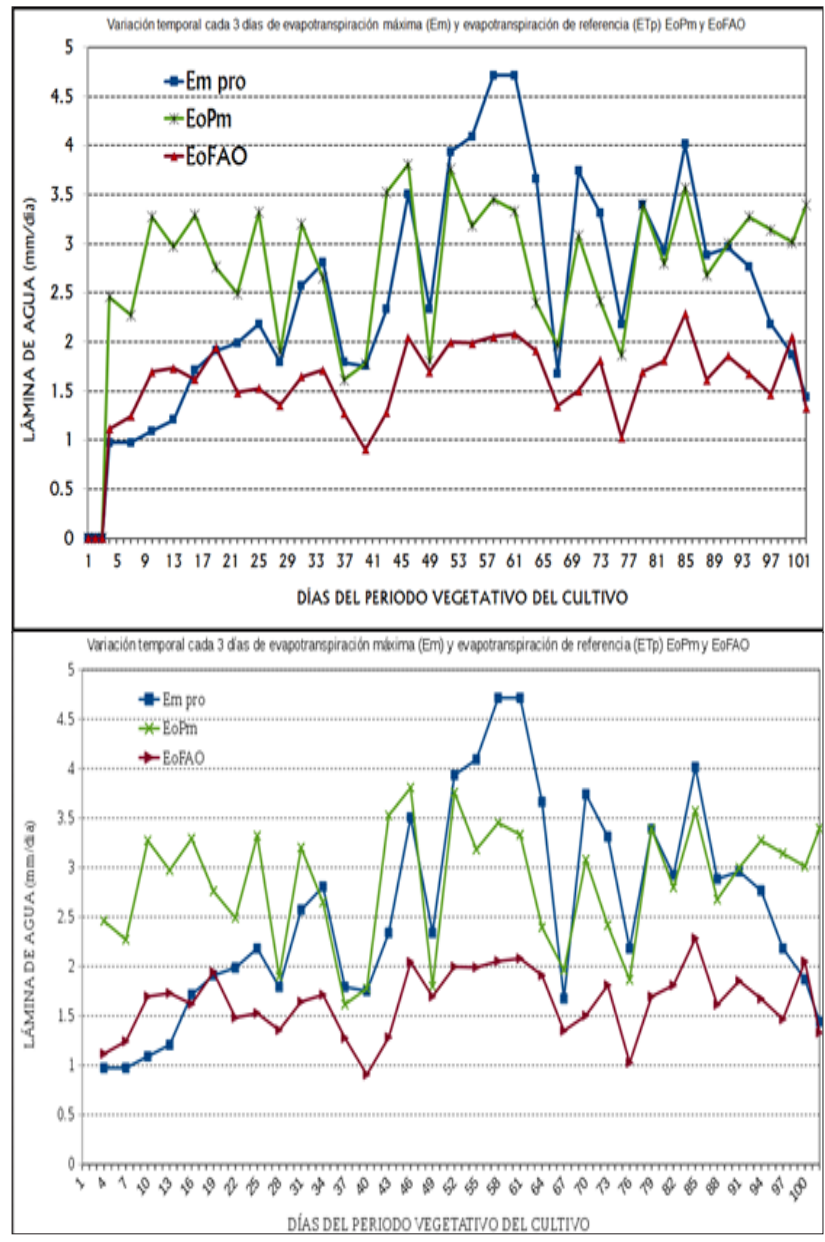

Figura 2. Evapotranspiración máxima promediado cada 3 días (Em pro) y evapotranspiración de referencia (ETo) promediado cada 3 días Penman-Monteith (EoFAO) y Penman Modificado para Perú (EoPm).

Tabla 3. Evapotranspiración máxima del cultivo de quinua QLM01 por etapas y total

\begin{tabular}{cccc}
\hline Periodo & Etapa & Em $(\mathrm{mm})$ & Em Total $(\mathrm{mm})$ \\
\hline $22 / 8-14 / 9$ & I & 37.7 & \\
$15 / 9-8 / 10$ & II & 58.8 & \\
$9 / 10-4 / 11$ & III & 95.73 & 262.3 \\
$5 / 11-30 / 11$ & IV & 69.9 & \\
\hline
\end{tabular}

\section{Determinación del coeficiente Kc del cultivo}

El coeficiente Kc del cultivo de quinua determinado se presenta en la Figura 3 y 4 . La Figura 3 muestra su variación temporal diaria, similar a la demanda hídrica los valores aumentan desde el inicio hasta la etapa de mediados de temporada ( $100 \%$ de desarrollo vegetativo) a partir de ella empieza su disminución, los valores son oscilantes en ambos métodos. La Figura 4 es la variación temporal de promedios de cada tres días, con ello se ha reducido las oscilaciones, se observa que Kc FAO oscila 
entre 0.69 en la etapa inicial y 2.48 en la etapa de media temporada, mientras que Kc-PeMod lo hace entre 0.33 en la etapa inicial y 1.52 en la etapa de media temporada; considerando los rangos extremos de Kc para cultivos completamente desarrollados fluctúa entre 1 y 1.4 (Allen et al., 2006), vemos que el método Penman-Monteith sobre estimó demasiado el Kc.

Teniendo en cuenta que en los estudios del Kc siempre se presentan valores promedios para cada una de las 4 etapas del cultivo, los Kc promedios para esas etapas del cultivo de quinua de esta investigación se presenta en la Tabla $\mathrm{N}^{\circ}$ 4. Allen et al. (2006) indican rangos típicos de Kc para cada una de las 4 etapas de crecimiento del cultivo, esos rangos son 0.2-1 etapa inicial, 0.3-1.25 etapa se desarrollo, 0.9-1.25 mediados de temporada y 1.25-0.2 en la etapa final, de acuerdo a estos valores Kc-FAO están fuera de esos rangos en las 4 etapas, mientras que Kc-PeMod están dentro de esos rangos.

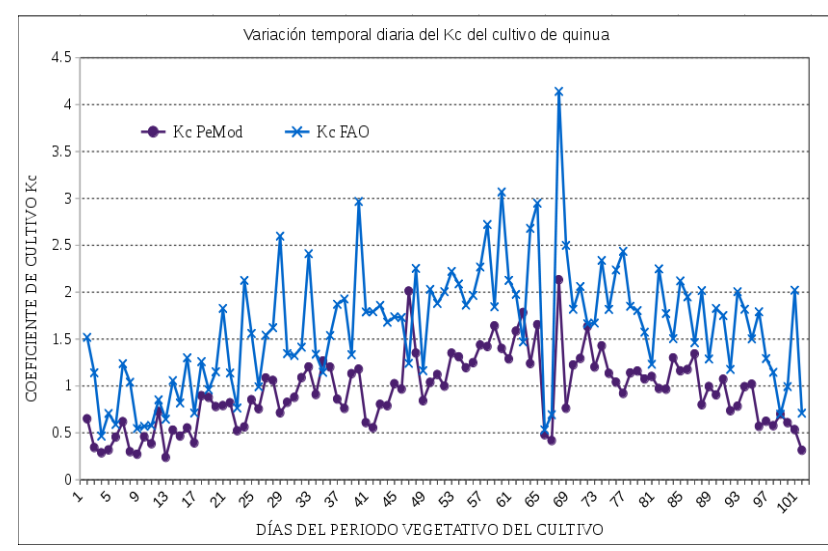

Figura 3. Variación temporal diaria del Kc del cultivo de quinua, determinado con demanda hídrica lisimétrica y evapotranspiración de referencia de Penman-Monteith (Kc FAO) líneas de asteriscos y Peman modificado para Perú (Kc PeMod) línea de puntos

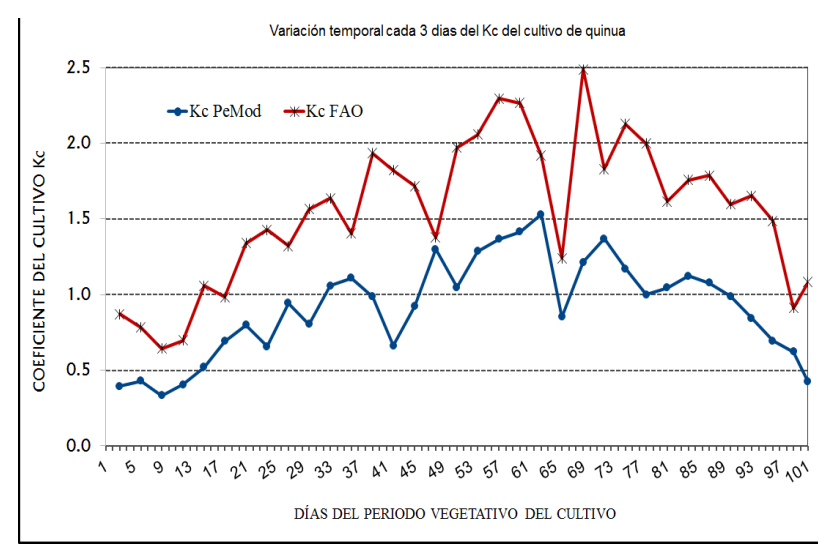

Figura 4. Variación temporal del Kc del cultivo de quinua promediado cada 3 días, determinado con demanda hídrica lisimétrica y evapotranspiración de referencia de PenmanMonteith (Kc FAO) línea de asteriscos y Peman modificado para Perú (Kc PeMod) línea de puntos
Tabla 4. Coeficiente Kc promedio por etapas del cultivo de quinua. Método Penman-Monteith (Kc FAO), Penman

\begin{tabular}{clcc}
\hline Periodo & \multicolumn{1}{c}{ Etapa } & Kc FAO & $\begin{array}{c}\text { Kc } \\
\text { PeMod }\end{array}$ \\
\hline $22 / 8-14 / 9$ & I (Inicial) & 1.022 & 0.554 \\
$15 / 9-8 / 10$ & II (Desarrollo) & 1.699 & 0.99 \\
$9 / 10-4 / 11$ & III (Mediados de temporada) & 2.112 & 1.271 \\
$5 / 11-30 / 11$ & IV (Final) & 1.581 & 0.904 \\
\hline
\end{tabular}

La Figura 1, muestra valores de ETo Penman-Monteith menores de $3 \mathrm{~mm} /$ día, los cuales comparados con Em cuyos valores llegan en el orden de $5 \mathrm{~mm}$ /día hace pensar que los ETo fueron subestimados, la razón de ello debe ser los datos de viento que no son del lugar del experimento puesto que se utilizaron de la estación Von Humboldt, la gran oscilación temporal de la evapotranspiración de la Figura indica alta turbulencia del viento y los datos de viento utilizados no indican ese comportamiento. Esto indica lo ideal de tener una estación meteorológica en el campo experimental o muy próximo al mismo.

\section{Conclusiones}

El uso del lisímetro de nivel freático constante permitió determinar la demanda hídrica (Em) del cultivo de quinua el cual fue $957.3 \mathrm{~m}^{3} /$ ha en la etpa de media temporada $\mathrm{y}$ $2623 \mathrm{~m}^{3} /$ ha durante la campaña agrícola.

La evapotranspiración máxima (Em) del cultivo determinado con el lisímetro y la evapotranspiración de referencia (ETo) determinados con el método de Penman adapatado para el Perú permitió determinar el Kc diario del cultivo cuyos valores oscilaron entre 0.33 y 1.52 , los que dieron los Kc promedios de $0.554,0.99,1.271$ y 0.904 en las etapas inicial, desarrollo, mediados de temporada y la etapa final respectivamente.

\section{Literatura citada}

Allen, R.; Pereira, L.; Raes, D. y Smith, M. 2006. Evapotranspiración del cultivo. Guías para la determinación de los requerimientos de agua de los cultivos. FAO, Roma.

Choquecallata, J.; Vacher, J.; Fellman T. y Imaña, E. 1991. evapotranspiración máxima del cultivo de quinua por lisimetría y su relación con evapotranspiración potencial en el Altiplano Boliviano. Actas del VII Congreso Internacional sobre Cultivos Andinos. La Paz, Bolivia. Pág. 66.

Garcia, V. 1992. Agrometeorología: Energía y Agua en la Agricultura. Ediciones UNALM.

Geerts, S. 2008. Dissertationes de Agricultura "Deficit irrigation satrtegies via crop wáter productivity modeling: Field research of quinoa in the Bolivian Altiplano". Doctoraatsproefschrift nr.814 aan de faculteit Bio-ingenieruswetenschappen van de K.U. Leuve. Bélgica. Disponible en: http://laquinua.blogspot. pe/2012/08/coeficiente-unico-del-cultivo-kc-de.html.

IICA. 2015. Informe anual 2015: Agricultura, oportunidad 
de desarrollo en las Américas / Harvesting results: annual report 2015. Disponible en: http://www.iica.int/es/ publications/informe-anual-2015-del-iica-agriculturaoportunidad-de-desarrollo-en-las-am\%C3\%A9ricas.

Mori, R. Allien. 2015. Efecto de cinco láminas de riego en el cultivo de quinua mediante el riego por goteo. Tesis de Ing. Agrícola.

Mujica; Jacobsen, S.; Izquierdo, J. y Marathee, J. 2001. Quinua, ancestral cultivo andino, alimento del presente y futuro. FAO, Oficina Regional de Producción vegetal para América Latina y el Caribe.

SENASA. 2014. Guía de Buenas prácticas Agrícolas para el cultivo de quinua. Disponible en: http://www.senasa. gob.pe/senasa/wp-content/uploads/2014/12/GUIABPA-QUINUA.pdf Revisado 24/04/17.

\section{Anexo \\ Implementació}

El lisímetro debe instalarse dentro del campo de cultivo cuya pérdida de agua se desea cuantificar, de modo que el tanque de cultivo esté rodeado del mismo cultivo con la finalidad de asegurar la misma exposición a los factores de evapotranspiración.

Debe evitarse en lo posible de los efectos de borde del campo de cultivo.

Por lo general debe ubicarse a sotavento del campo de cultivo para garantizar el requisito anterior (Figura 5)

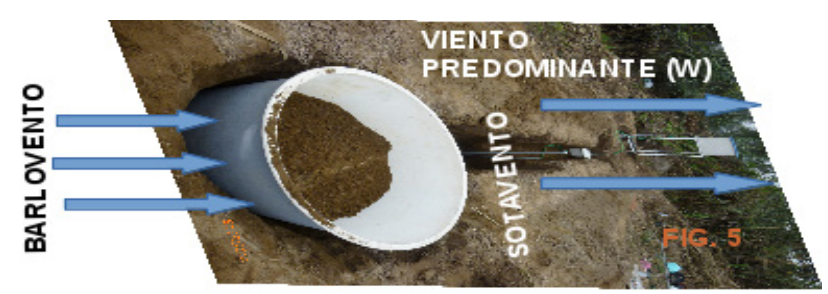

Figura 5. Ubicación del tanque de cultivo del lisímetro en sotavento del campo experimental.

\section{Instalación del lisímetro}

Antes de realizar cualquier actividad agrícola en el campo a ser sembrado con el cultivo cuyo consumo de agua se desea cuantificar, primero debe realizarse varias calicatas para determinar la humedad en capacidad de campo, esta información será utilizada en la calibración del lisímetro. La determinación de humedad en capacidad de campo se verá posteriormente.

Una vez fijado el emplazamiento del equipo del lisímetro se procederá a su instalación como se indica a continuación:

- $\quad$ El tanque de cultivo se ubica aproximadamente a 12 $\mathrm{m}$ de distancia del borde del campo agrícola.

- El tanque controlador de nivel freático se ubica en el borde o frontera del campo agrícola a una profundidad de unos 70 a $80 \mathrm{~cm}$.

- El tanque alimentador se ubica sobre un soporte de $1.3 \mathrm{~m}$ de altura a una distancia de $50 \mathrm{a} 60 \mathrm{~cm}$ del tanque controlador del nivel freático.

En la instalación del tanque de cultivo, al realizar la excavación, se debe tener en cuenta las siguientes consideraciones:

- Retirar primera capa (arable) de $30 \mathrm{~cm}$, colocarlo haciendo un montículo.

- Retirar la segunda capa de $40 \mathrm{~cm}$, colocarlo haciendo un montículo bien separado del primero.

- Finalizar retirando la capa restante de $50 \mathrm{~cm}$, colocando similar a los anteriores bien separados de los dos ya existentes.

- $\quad$ Luego continuar con la excavación de la zanja de 1.2 $\mathrm{m}$ de profundidad por tres metros de longitud contiguo al tanque de cultivo. 
- Completar la excavación de la zanja de 50 a 60 cm de profundidad hasta la ubicación del tanque controlador del nivel freático.

\section{Nivelación y conexión de los tanques}

Una vez concluida con la excavación de las zanjas, se proceden a colocar los tanques en sus posiciones respectivas bien nivelados horizontalmente. Luego se procede a su conexión con las tuberías y mangueras, como se indica en la Figura 6. Una vez conectado los tres tanques deben completarse colocando la boya y la válvula en el tanque controlador del nivel freático y la pipeta en el tanque alimentador.

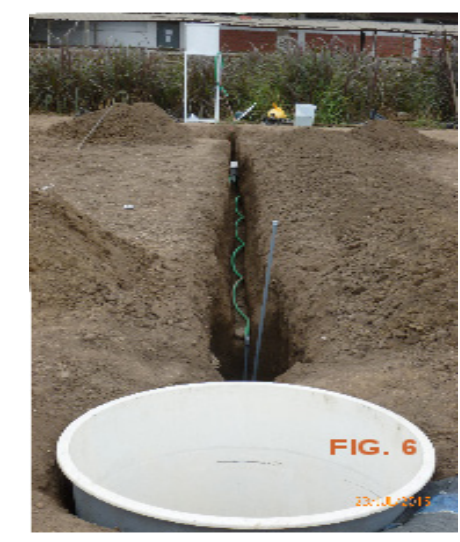

Figura 6. Tanques del equipo de lisímetro conectados y nivelados.

Una vez concluida con las conexiones de los tanques, el siguiente paso es ver que las conexiones realizadas no permitan ninguna filtración o fuga de agua en especial poner atención en la conexión de la tubería con el tanque de cultivo y la unión universal (Figura6).

La prueba de ausencia de filtración se realiza llenando un poco de agua en el sistema o equipo de lisímetro por un periodo de 8 a 12 horas.

Una vez verificado la ausencia de filtraciones, retirar el agua del sistema para continuar con la implementación del equipo de lisímetro.

\section{Llenado del tanque de cultivo}

El tanque de cultivo es la parte más importante de todo equipo de lisímetro, consta de dos partes principales, la parte inferior conformado por sub capas de piedra y otra capa superior de suelo de $80 \mathrm{~cm}$ de espesor aproximadamente. Para la conformación de esas capas seguir el siguiente procedimiento:

- Realizar las marcas a $30 \mathrm{~cm}, 70$ y $90 \mathrm{~cm}$ de profundidad, las cuales serán el tope superior de capa de piedra, tercera capa (la más profunda) y segunda capa de suelo respectivamente.

- Formar las sub capas de piedra empezando con canto rodado más grandes, seguido por confitillo o grava y finalizando con piedra chancada hasta la señal de 90 $\mathrm{cm}$.

- Devolver la tercera capa de suelo compactar con cuidado hasta alcanzar la señal de $70 \mathrm{~cm}$.

- Devolver la segunda capa de suelo hasta la señal de $30 \mathrm{~cm}$ realizando proceso de compactación ligera.

- Completar con la devolución de la primera capa de suelo. El resultado del llenado del tanque de cultivo se verá como se observa en la Figura 7.

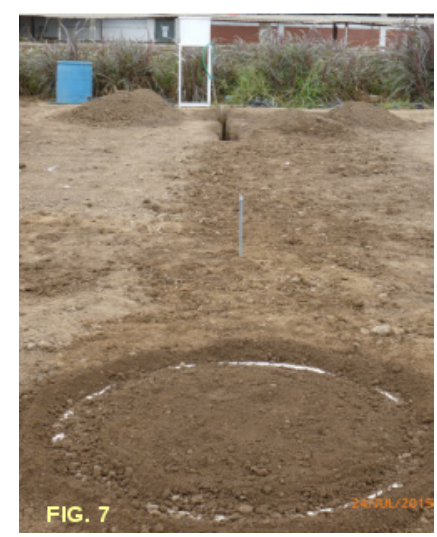

Figura 7. Equipo de lisímetro instalado listo para la calibración.

\section{Calibración del lisimetro}

Para la calibración del equipo es necesario contar con humedad del suelo en base a peso en su capacidad de campo para las profundidades de $10,20,30$ y $40 \mathrm{~cm}$. Para determinar esta humedad se hace necesario la humedad volumétrica en capacidad de campo y la densidad global.

\section{Determinación de humedad $\theta_{\mathrm{CC}}$, densidad global $\rho_{\mathrm{g}} \mathrm{y}$ humedad gravimétrica $\mathbf{u}_{\mathbf{C C}}$}

Una vez lista las calicatas se toman muestras en por lo menos 4 niveles de profundidad (Z1, Z2, Z3 y Z4), abarcando desde $2 \mathrm{~cm}$ hasta 40 ó $50 \mathrm{~cm}$ de profundidad, tratando de cubrir la profundidad de las raíces del cultivo en su etapa de $100 \%$ desarrollo vegetativo.

Con las muestras obtenidas se determinan el peso húmedo $(m h)$, se coloca en estufa a $105^{\circ} \mathrm{C}$ hasta alcanzar peso constante, obteniéndose así el peso seco $(\mathrm{ms})$. Con ello la humedad volumétrica $\theta_{\mathrm{CC}}$ está dada por:

$$
\theta_{c \mathrm{C}}=\frac{\mathrm{mh}-\mathrm{ms}}{\mathrm{V}}=\frac{\text { Vagua }}{\text { Vsuelo }}\left(\frac{\mathrm{cm}^{3}}{\mathrm{~cm}^{3}}\right)
$$

Densidad global $\left(\rho_{g}\right)$ : Es la masa $(m s)$ de muestra de suelo existente en el volumen $(V)$ que ocupa dicha muestra, o $\rho_{\mathrm{g}}=\frac{\mathrm{ms}}{\mathrm{V}}\left(\frac{\mathrm{gr}}{\mathrm{cm}^{3}}\right)$

Humedad en base a peso $\left(u_{C} \boldsymbol{C}\right)$ : Este parámetro físico 
representa el valor promedio de una capa de suelo por ello su cálculo en función de $\theta_{\mathrm{cc}}$ y $\rho_{g}$ está dada por:

$$
\mathrm{u}_{c c}=\frac{\theta \mathrm{cc}}{\overline{\mathrm{P}}_{\mathrm{ge}}}\left(\frac{\mathrm{gr}}{\mathrm{g}}\right)
$$

En la cual la humedad volumétrica promedio en dicha capa está dada por:

$$
\bar{\theta} \mathrm{cc}=\frac{(\theta \mathrm{cc}) 1+(\theta \mathrm{cc}) 2+\cdots+(\theta \mathrm{cc}) \mathrm{n}}{\mathrm{n}}
$$

De manera similar, la densidad global específica está dado por:

$\bar{\rho}_{g e}=\frac{\left(\rho_{g}\right) 1+\left(\rho_{g}\right) 2+\ldots+\left(\rho_{g}\right) n}{\rho_{\text {agua }} n}$

En las expresiones anteriores tanto la humedad volumétrica $(\theta \mathbf{c c})$ como la gravimétrica $\left(\mathbf{u}_{\mathbf{c c}}\right)$ están expresadas en porcentaje o en forma a dimensional; sin embargo para su aplicación en riego de cultivos es necesario expresarlos como lámina de agua, cuyo procedimiento se ve a continuación.

\section{Humedad volumétrica $\theta$ cc y gravimétrica $\mathbf{u}_{\mathrm{CC}}$ como lámina de agua}

La lámina de agua en el suelo, en cuanto se conoce la humedad porcentual, depende de la profundidad o capa de suelo en consideración. Si esa capa es la profundidad del sistema radicular $(\mathrm{Zr})$ de la planta, entonces la lámina de agua aproximada hasta la profundidad $\mathrm{Zr}$ está dada por la expresión:

$g_{Z r}=\bar{\theta}_{c c} \cdot Z r=\left(\frac{\theta_{c c} 1+\theta_{c c} 2+\cdots+\theta_{c c} n}{n}\right) \cdot Z r$

En casos de humedad en base a peso (u) su expresión está dada por:

$g_{\mathrm{Zr}}=\bar{\rho}_{\rho \varepsilon} \bar{u} \cdot \operatorname{Zr}(\mathrm{mm})$

Ejemplo de expresar la humedad volumétrica $\theta$ cc como lámina de agua

Consideremos, al realizar una calicata, se han tomado muestras a $2,15,25$ y $40 \mathrm{~cm}$ de profundidad, con ello se han obtenido $\Theta c c(\%)$ y $D g\left(\mathrm{~g} / \mathrm{cm}^{3}\right)$ que se muestran a continuación:

\begin{tabular}{ccccc}
\hline Nivel (cm) & Occ (\%) & Dg $\left(\mathrm{g} / \mathrm{cm}^{3}\right)$ & $\mathrm{g}_{\mathrm{Zr}}(\mathrm{mm})$ & $\mathrm{U}(\%)$ \\
\hline 2 & 31.13 & 1.25 & $\mathrm{x}$ & $\mathrm{x}$ \\
15 & 32.23 & 1.38 & $\mathrm{x}$ & $\mathrm{x}$ \\
25 & 32.18 & 1.46 & $\mathrm{x}$ & $\mathrm{x}$ \\
40 & 30.46 & 1.63 & $\mathrm{x}$ & $\mathrm{x}$ \\
\hline
\end{tabular}

Las láminas de agua para las capas 0-2, 0-15, 0-25 y $0-40 \mathrm{~cm}$ o $\mathrm{Zr}$ de $2,15,25$ y $40 \mathrm{~cm}$ de profundidad son las siguientes:

\begin{tabular}{ccccc}
\hline $\mathrm{Zr}(\mathrm{cm})$ & $\Theta \mathrm{cc}(\%)$ & $\mathrm{Dg}\left(\mathrm{g} / \mathrm{cm}^{3}\right)$ & $\mathrm{g}_{\mathrm{Zr}}(\mathrm{mm})$ & $\mathrm{U}(\%)$ \\
\hline 2 & 31.13 & 1.25 & $\mathbf{6 . 2 3}$ & $\mathbf{2 4 . 9 0}$ \\
15 & 31.68 & 1.32 & $\mathbf{4 7 . 5 2}$ & $\mathbf{2 4 . 0 0}$ \\
25 & 31.85 & 1.36 & $\mathbf{7 9 . 6 3}$ & $\mathbf{2 3 . 4 2}$ \\
40 & 31.5 & 1.43 & $\mathbf{1 2 6 . 0 0}$ & $\mathbf{2 2 . 0 3}$ \\
\hline
\end{tabular}

En la cual la última columna es la humedad gravimétrica y son las necesarias para la calibración del lisímetro.

\section{Llenado de agua al equipo del lisímetro}

El proceso de calibración demora de 3 a 4 semanas, empieza con el llenado de agua, a través del tanque alimentador, al equipo de lisímetro; el llenado es rápido hasta que la lámina de agua alcance la capa de suelo. Después de esto el consumo o ingreso de agua es lento.

El tanque controlador del nivel freático debemos fijar de tal forma que la lámina de agua alcance la altura de 68 a $70 \mathrm{~cm}$.

Después de fijar el nivel freático, el agua sube a la superficie por capilaridad y el proceso es lento y puede demorar varios días; mientras eso ocurre debemos tener cuidado con el tanque alimentador, se debe reponer agua hasta el nivel de $0 \mathrm{ml}$ toda vez que se aproxime el nivel a los $40 \mathrm{ml}$. Nunca debe esperarse que se consuma todo el agua de este tanque (Figura 8).

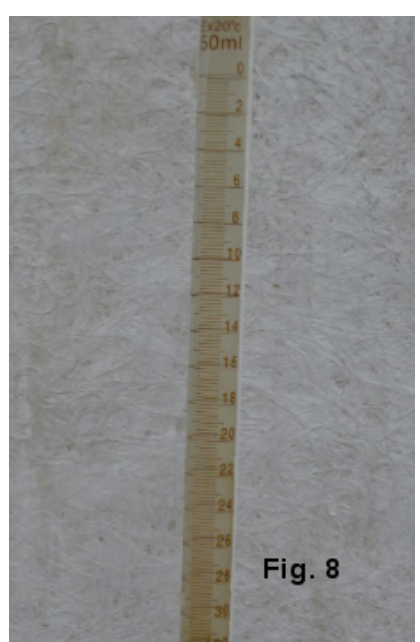

Figura 8. Sistema de lectura del lisímetro

\section{Muestreo de humedad de suelo del tanque de cultivo}

En cuanto la humedad haya llegado a la superficie debemos esperar 2 a 3 días para que el suelo húmedo se afirme y compacte para la toma de muestra.

La toma de muestra se realizará para $\mathrm{Zr}$ de 15,25 y $35 \mathrm{~cm}$ de profundidad, utilizando para ella el tornillo muestreador. Se deben tomar como mínimo tres repeticiones; obtener los pesos húmedos $(\mathrm{mh})$, colocar en estufa hasta adquirir 
peso constante obteniendo con ello los pesos secos (ms).

Con la información disponible determinar la humedad gravimétrica para cada Zr. Luego comparar con los correspondientes a humedad en capacidad de campo previamente determinado.

Es importante indicar que es necesario aplicar los datos de capacidad de campo a los datos de $\operatorname{Zr}(15,25$ y $35 \mathrm{~cm})$ para poder realizar la comparación.

Si los resultados del muestreo son mayores a los de capacidad de campo, se procede a bajar el nivel freático, en caso contrario se tiene que subir; luego esperar una semana para realizar el nuevo muestreo. Ese proceso se debe repetir hasta conseguir que la humedad en el tanque de cultivo se iguale a la de capacidad de campo. De ser así, el equipo ya está calibrado y listo para la siembra del cultivo y su operatividad. En este experimento se logró fijar el nivel freático o capa de lámina de agua a $67 \mathrm{~cm}$.

\section{Siembra del cultivo en el lisimetro}

Una vez calibrado el equipo de lisímetro, se procede a sembrar el cultivo cuyo consumo de agua se desea conocer y debe realizarse en forma simultánea con el campo de cultivo de su entorno (Figura 9).

En la siembra debe mantenerse la misma densidad de siembra como en el campo de cultivo.

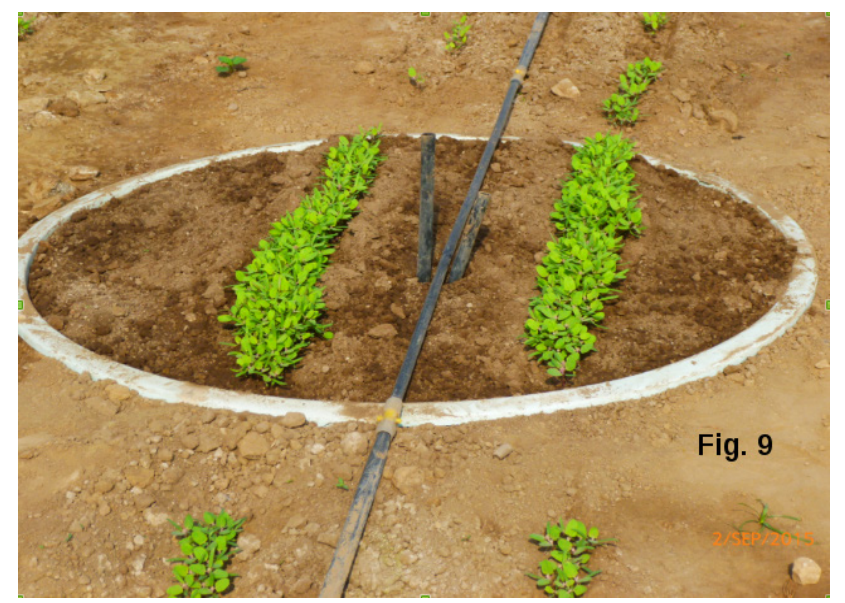

Figura 9. Cultivo de quinua a 11 días después de la siembra

\section{Funcionamiento u operación del lisimetro}

El funcionamiento del lisímetro consiste en la cuantificación de la evapotranspiración total diaria del cultivo. Para ello se deben realizar lecturas de la pipeta a las 7 a.m y 7 p.m. llenando el formato preparado para ese fin.

La evapotranspiración se obtiene de la diferencia (L) de una lectura final (Lf) menos una lectura inicial (Li). Como las lecturas están expresadas en $m l$ la diferencia también está en $\mathrm{ml}$. Para obtener la evapotranspiración(Em) en $\mathrm{mm}$ se debe multiplicar por un factor de conversión. El factor de conversión es propio de cada equipo de lisímetro. Las Figuras 10 y 11 muestran etapas de desarrollo a 36 días y 68 días después de la siembra.

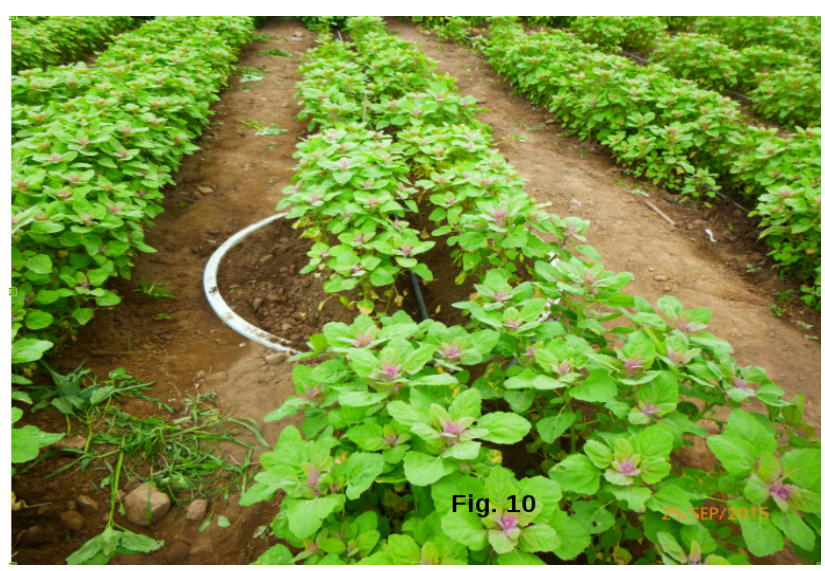

Figura 10. Cultivo de quinua a 36 días después de la siembra

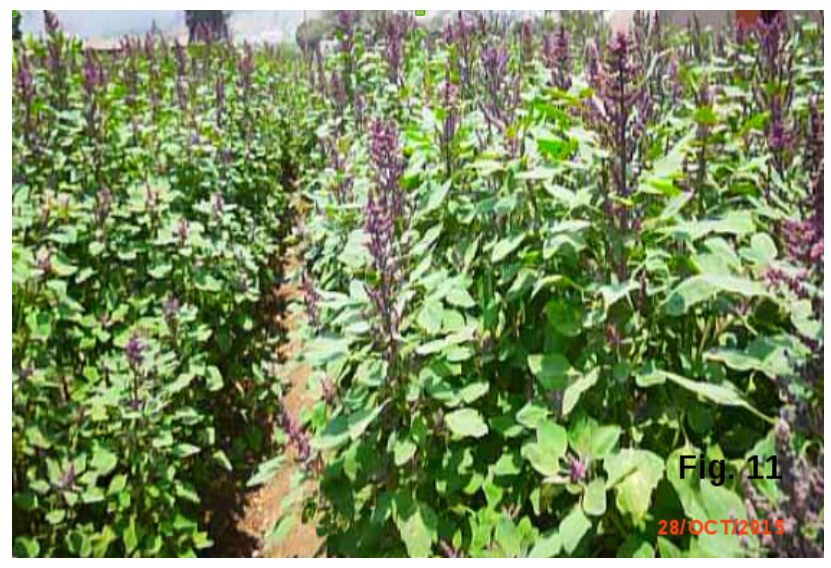

Figura 11. Cultivo de quinua a 68 días después de la siembra 